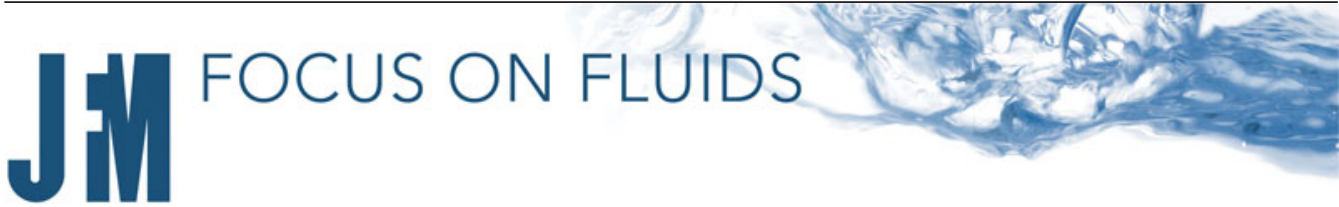

\section{The Saturnian droplet}

\author{
A. Marin $\dagger$ \\ Physics of Fluids, University of Twente, 7500 AE Enschede, The Netherlands
}

(Received 6 November 2020; accepted 9 November 2020)

Electrohydrodynamic instabilities at liquid interfaces continue to defy our intuition, from the pioneering work of Taylor (Proc. R. Soc. Lond. A, vol. 280, issue 1382, 1964, pp. 383-397) on conical tips of electrified droplets to a recent numerical study by Wagoner et al. (J. Fluid Mech., vol. 904, 2020, R4). The problem studied by Wagoner et al. (2020) consists of a droplet immersed in a more conducting and more dielectric liquid medium, in a strong electrical field. When the droplet is more viscous than the outer medium, the droplet develops a biconcave shape which might eventually evolve to a torus shape (or doughnut). In contrast, when the droplet is less viscous, it adopts a lenticular shape and emits a thin fluid sheet from its equator which in turn breaks up into droplets. These droplets form a ring of satellites around the original droplet, which justifies its appellation 'Saturnian droplet'. The numerical simulations bring light to this complex phenomenon and confirm the robustness of the leaky-dielectric framework (Melcher \& Taylor, Annu. Rev. Fluid Mech., vol. 1, 1969, pp. 111-146).

Key words: drops, electrohydrodynamic effects, drops and bubbles

\section{Introduction}

Our intuition stems from our everyday experiences. Even though we cannot observe gravitational field lines, we can infer the trajectory of a falling apple under its own weight. Unfortunately, this same intuition does not work well for electromagnetic fields, because their interaction with matter is not part of our everyday experience. Electrohydrodynamics (EHD), which results from the interaction of electromagnetic fields and liquid matter, is even less in tune with our intuition.

This field dates back to the seventeenth century, when an English physician and pioneering experimental physicist, William Gilbert, briefly described in his work de Magnete (1600) how rubbed amber can exert attraction to a water droplet, deforming it into a conical shape. Lord Rayleigh (1882) determined theoretically the maximum amount of electrical charge that a droplet can hold before becoming unstable. We had to wait

$\dagger$ Email address for correspondence: a.marin@utwente.nl

(C) The Author(s), 2020. Published by Cambridge University Press. This is an Open Access article, distributed under the terms of the Creative Commons Attribution licence (http://creativecommons.org/ licenses/by/4.0/), which permits unrestricted re-use, distribution, and reproduction in any medium, provided the original work is properly cited. 


\section{A. Marin}

until the 20th century for controlled electrohydrodynamic experiments to be performed, in particular on imperfect conductors which encompass most liquids in nature. For example, the experimental work of Mason (Allan \& Mason 1962; Torza et al. 1971) systematically revealed a plethora of hidden phenomenology. Shortly afterwards came the acclaimed work of Taylor in this field: first on conical singularities (Taylor 1964) and then, together with James Melcher, their celebrated leaky-dielectric model (Melcher \& Taylor 1969), which established a very solid ground to study the complexity revealed by experiments.

The work of Taylor on conical tips stabilized by EHD stresses served as a basis to understand more complex EHD phenomena like electrospray (Fernández De La Mora 2007). This became a standard technique for atomization, used extensively in mass spectrometry, and it was the subject of the 2002 Nobel Prize in Chemistry awarded to Fenn et al. (1989). Recently, Brosseau \& Vlahovska (2017) found an instability, coincidentally also briefly mentioned by Torza et al. (1971), with several analogies to EHD tip streaming. While pointy tips (Taylor cones) develop when conducting droplets are exposed to intense electrical fields in a non-conducting medium, the modes uncovered by Brosseau \& Vlahovska (2017) occur with liquid droplets surrounded by a more conducting and more dielectric medium. Under these conditions, depending on the viscosity contrast with the external medium, the droplet adopts either a biconcave or a lenticular shape when placed in a strong, uniform electric field. The instabilities can grow further with discocyte-shaped droplets eventually evolving into tori. Conversely, a lenticular droplet might develop sharp edges; not at the poles of the droplet, but at its equator. If the electrical field is strong enough, a thin sheet emanates from the droplet's equator, which quickly breaks into smaller droplets. This striking phenomenon is referred to as equatorial streaming, in contrast with the better known tip streaming.

One of the most important questions to answer is whether the theoretical framework left by Taylor, the leaky-dielectric model (Melcher \& Taylor 1969), can explain the two extreme modes observed, the biconcave (discocyte) and the lenticular droplet, with the latter undergoing equatorial streaming as a Saturnian droplet. Do these two instabilities arise due to the same mechanisms? The complexity of the problem requires a careful numerical and theoretical analysis, which has been successfully achieved by Wagoner et al. (2020) and is the purpose of this short review.

\section{Overview}

In their paper, Wagoner et al. (2020) solved the leaky-dielectric equations for two of the extreme viscosity ratios explored experimentally by Brosseau \& Vlahovska (2017). To do so, Wagoner et al. (2020) solved the equations with a finite-element method, using an adaptive parametrization to search systematically for steady-state solutions and identify changes of stability in the solutions. With this method, the authors mapped droplet stability with bifurcation diagrams of stable droplet shapes for different levels of electrification (see figure 1).

The flow direction in both phases can be readily predicted by analysing the leaky-dielectric model equations for low electrical fields following Melcher \& Taylor (1969), which concluded that electrical stresses at the poles are always compressive, and electrical shear stresses drive flow from the poles to the equator. This is, however, not enough to explain the different modes found. Focusing solely on asymptotic cases (perfect conductor/insulator or limits of viscosity) does not provide the additional information required, because electrical shear and normal hydrodynamic stresses are not important in these limits. The numerical results of Wagoner et al. (2020) show how the different interfacial stresses play different roles in different families of steady-state solutions. 


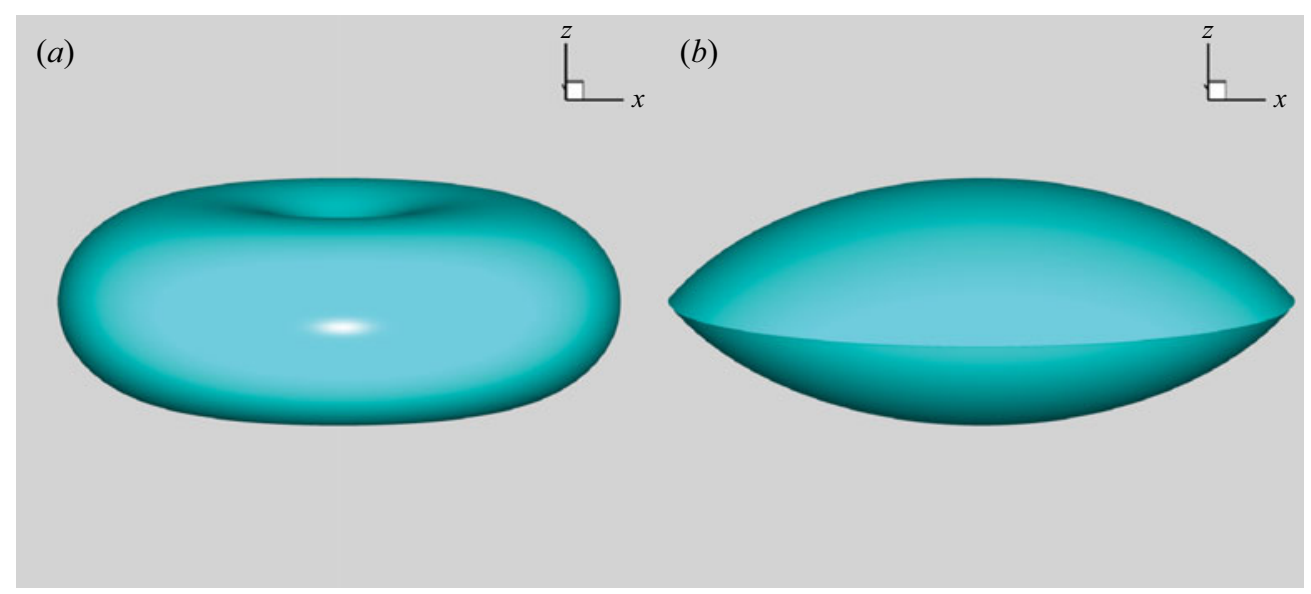

Figure 1. Drop shapes obtained from steady-state solutions of the leaky-dielectric equations by Wagoner et al. (2020) for $(a) \mu_{\text {medium }}=0.02 \mu_{\text {drop }}$, showing a biconcave/discocyte shape and for $(b) \mu_{\text {medium }}=50 \mu_{\text {drop }}$ showing the lenticular shape which eventually leads to equatorial streaming.

The biconcave shapes (see figure 1a) develop entirely due to the presence of electrical shear stresses, with a marginal influence of normal electrical stresses. As the electric field and the electrical shear stress increases, so does the interfacial flow, increasing the pressure at the equator and decreasing it at the poles, causing discocyte-shaped droplets. To prove this point, the electrical normal stresses can be turned off, and a comparable family of stable solutions with discocyte shapes is obtained.

Surprisingly, the role of electrical stresses changes completely when the viscosity ratio is switched so that the droplet is less viscous than the external medium. In this case, the flow in the more viscous external medium is drawn towards the drop's poles and pushed from its equator. In the absence of electrical normal stresses, only stable oblate shapes are found. However, the family of stable solutions changes dramatically when electrical normal stresses are turned on: these counteract the effect of the capillary and hydrodynamic stresses and stabilize the droplet deformation for increasing electrification. Locally at the equator, although the stresses balance, they increase with electrification, thus generating stronger flows, sharper curvatures at the equator and leading to lenticular shapes (see figure $1 b$ ). Such a dramatic equilibrium of interfacial stresses shares similarities, but also important differences, with the stability of Taylor cones, which results from a balance between electric normal stresses and surface tension. Such a balance is eventually broken by electrical shear stresses into EHD tip streaming (Collins et al. 2013) or electrosprays (Fernández de La Mora \& Loscertales 1994). In summary, these important results shed light on the complex phenomena initially spotted by Torza et al. (1971), then carefully studied experimentally by Brosseau \& Vlahovska (2017), and confirm the strength of the framework developed by Melcher \& Taylor (1969).

\section{Future}

There is still work ahead: the results of Wagoner et al. (2020) are limited to steady-state solutions and a proper description of the instability process is still lacking. In the same way steady conical droplet shapes were explained by Taylor (1964), but it took years to understand how they become unstable and eject their charge and mass according to precise scaling laws (Fernández de La Mora \& Loscertales 1994). Time-resolved simulations 


\section{A. Marin}

of these EHD instabilities are challenging because of the large disparities in length and time scales: from the millimetric size of the drop, to the micrometric size of the ejected film and droplets; from the slow deformation of the drop on the scale of a second, to fast film formation and consequent breakup on millisecond scales. Nonetheless, the time evolution of an EHD system of similar complexity to electrohydrodynamic tip streaming has been recently computed numerically (Collins et al. 2008, 2013), and we expect that similar methods could be applied to the Saturnian droplet to shed light on their mass and charge transfer process. But there is also work ahead for experimentalists: the equatorial streaming needs to be further investigated. For example, a comparison between experiments and numerical simulations could be done through the flow field around the droplet, but experimental data in this aspect are still missing. Additionally, the results of Wagoner et al. (2020) have identified an interesting hysteresis for biconcave droplets, which resembles that found numerically for dielectric sessile droplets, but has not yet been observed experimentally.

A better understanding of the phenomenon could lead to potential applications of the Saturnian droplet as an emulsification technique, covering a range of parameters that submerged electrosprays (Marin et al. 2007) cannot reach, and without the local hydrodynamic stresses required for hydrodynamic tip streaming (Suryo \& Basaran 2006).

Declaration of interests. The author reports no conflict of interest.

Author ORCIDs.

(1) A. Marin https://orcid.org/0000-0002-5586-2025.

\section{REFERENCES}

Allan, R.S. \& Mason, S.G. 1962 Particle behaviour in shear and electric fields. I. Deformation and burst of fluid drops. Phil. Trans. R. Soc. Lond. A 267 (1328), 45-61.

BRosseau, Q. \& VLAhovsKa, P.M. 2017 Streaming from the equator of a drop in an external electric field. Phys. Rev. Lett. 119 (3), 034501.

COllins, R.T., JoneS, J.J., HARRIS, M.T. \& BASARAN, O.A. 2008 Electrohydrodynamic tip streaming and emission of charged drops from liquid cones. Nat. Phys. 4 (2), 149-154.

Collins, R.T., Sambath, K., Harris, M.T. \& Basaran, O.A. 2013 Universal scaling laws for the disintegration of electrified drops. Proc. Natl Acad. Sci. USA 110 (13), 4905-4910.

Fenn, J.B., Mann, M., Meng, C.K., Wong, S.F. \& Whitehouse, C.M. 1989 Electrospray ionization for mass spectrometry of large biomolecules. Science 246 (4926), 64-71.

Fernández De La Mora, J. 2007 The fluid dynamics of Taylor cones. Annu. Rev. Fluid Mech. 39 (1), $217-243$.

FERnÁndez de La Mora, J. \& Loscertales, I.G. 1994 The current emitted by highly conducting taylor cones. J. Fluid Mech. 260, 155-184.

Marin, A., Loscertales, I.G., Márquez, M. \& Barrero, A. 2007 Simple and double emulsions via coaxial jet electrosprays. Phys. Rev. Lett. 98 (1), 014502.

Melcher, J.R. \& TAYLOR, G.I. 1969 Electrohydrodynamics: a review of the role of interfacial shear stresses. Annu. Rev. Fluid Mech. 1, 111-146.

RAYLEIGH, LORD 1882 XX. On the equilibrium of liquid conducting masses charged with electricity. Phil. Mag. 14 (87), 184-186.

SURYO, R. \& BASARAN, O.A. 2006 Tip streaming from a liquid drop forming from a tube in a co-flowing outer fluid. Phys. Fluids 18 (8), 082102.

TAYLOR, G.I. 1964 Disintegration of water drops in an electric field. Proc. R. Soc. Lond. A 280 (1382), 383-397.

ToRZA, S., Cox, R.G. \& MASON, S.G. 1971 Electrohydrodynamic deformation and bursts of liquid drops. Phil. Trans. R. Soc. Lond. A 269 (1198), 295-319.

Wagoner, B.W., Vlahovska, P.M., Harris, M.T. \& Basaran, O.A. 2020 Electric-field-induced transitions from spherical to discocyte and lens-shaped drops. J. Fluid Mech. 904, R4. 